\title{
EFFECT OF BIOFERTILIZER TRICHODERMA HARZIANUM T-22 APPLICATION, GROWING MEDIUM AND TRAINING METHODS ON SOME CHARARCTRESTICS FOR LANTANA CAMARA PLANTS
}

\author{
${ }^{1}$ Afnan B. Yahya ${ }^{2}$ Mohammed D. Al-Sawaf ${ }^{3}$ Nidal Y. Al-Morad \\ ${ }^{1,2}$ Horticulture and land scape Dept., ${ }^{3}$ Plant protection Dept ., College of Agriculture \\ and Forestry, Mosul University. Iraq \\ afnan.1992@uomosul.edu.iq
}

\begin{abstract}
An experiment was in the green net canopy Department of Horticulture and Landscape College of Agriculture and Forestry / University of Mosul from May 2019 to October 2019, to investigate the effect of Trichoderma harzianum T-22 application and three growing media (soil, 1:3 soil: peatmoss, 1:1 soil: peatmoss) and three pruning training method (1, 4, 6 branches) and their interaction on the vegetative and flowering growth of Lantana camara. Results showed that $T$. harzianum application, the growing medium, pruning training method and their interaction had significant effects on the vegetative and flowering growth of plant. The highest average of plant height, stem diameter, leaves number, chlorophyll content and flowering date gives in T. harzianum which increases plant height which was $52.31 \mathrm{~cm}$, stem diameter $8.06 \mathrm{~cm}$, leaves number 164.65, chlorophyll content 25.98 SPAD and flowering date 85.84 day. Results have shown that pruning training Lantana plants on six branches gave the highest average for plant height $52.29 \mathrm{~cm}$, chlorophyll content 38.69 SPAD and flowering date 79.25 day. Growing medium 1:1 soil: peat moss gives the highest average for plant height $51.97 \mathrm{~cm}$, stem diameter $8.28 \mathrm{~cm}$, chlorophyll content 26.29 SPAD and flowering date 93.33 day.
\end{abstract}

Keywords: Trichoderma harzianum T-22, Lantana camara, Growing media, peat moss, Training method.

Received: $10 \backslash 8 \backslash 2020$, Accepted: $30 \backslash 3 \backslash 2021$

\section{INTRODUCTION}

Lantana is an ornamental plant planted in garden and parks .The elegant floral colors of Lantana include white, yellow, blue, purple and red. Lantana plants do not grow well in shaded areas because lantana needs a location with high light intensity (Muthahara et al., 2018).

Growing media plays a key role in the quality of flowering plants and their development. Natural soil and peat are the most used growing substrates for annual and perennial ornamental plant production in containers. Using organic media rich in nutrients can potentially lead to a reduction in fertilization and irrigation levels, as well as to a reduction in nursery costs. Peat is the most widely used substratum for potted plant production in nurseries and represents a substantial part of the material used to grow potted plants (Younis et al., 2015). Growth medium is known to have been active in adding value to potted ornamental plants and should have the best characteristics such as good aeration, water retention potential and sufficient

The research is a part of M.Sc. thesis of the first researcher. 
supply of nutrients In addition, growth medium plays an important part in physiological parameters such as plant height, number of leaves, number and diameter of flowers .The optimum amount of nutrients and environmental factors have been reported to affect plant growth, development and flowering of petunia (Mehmood et al., 2013).

The purpose for pruning is to track and monitor plant growth to maximize the development of buds, flowers and fruits in various seasons or across the year. Many scholars and their experiments have emphasized the effects of flower pruning and its

The pruning technique is traditional and productive for a variety of ornamental Plants under natural open sunlight or greenhouse conditions.consequences for the subsequent growth of fruit their quantity. Yet still ornamental flowering plants, usually concentrated on initiating new flowering shooting by creating or increasing the flowering shoot .In the meantime, the fewer shoots develop in the unpruned plants may be induced a short-term Reduced cell activity of older shoots and caused yield drop manufacture, Therefore, in the long run growing stage, the plant's nutrient reserves cannot improve, moderately or completely, and retain the initiation of their flowers again. The level of recovery of the plants would therefore depend on pruning, in particular, pruning position, pruning height and the timing of plant phase (Saifuddin $e t$ al., 2010). Trichoderma are the most commonly used microorganisms with biocontrolled disease and activity-promoting plant growth Such fungi are mycoparasites that develop a variety of secondary antimicrobial compounds including phytohormones Phytohormones released by Trichoderma spp. Actually they are marketed as biopesticides, biofertilizers, increasing and rising yields as well as nutrient solubilizers and organic matter decomposers An interesting aspect of Trichoderma mediated biocontrol their ability to colonize roots and induce systemic resistance against invading fungi, bacteria, viruses and even insects, at a site away from Trichoderma inoculation (Swain et al., 2018).

\section{MATERIALS AND METHODS}

Throughout the year 2019 present work project was carried out at the Horticultural department, college of agriculture and forestry University of Mosul. A planted variety of Lantana was transplanted into the pots.

Biofertilizers: Trichoderma harzianum isolate T22, obtained from (CBS), Spore suspensions were adjusted to $8 \times 106 \mathrm{~mL}^{-1}$. Growing media: Three different growing media used in the pot experiment in which Lantana cuts are used are (soil, 1:1 soil: peat moos and 1:3 soil: peat moos). Pruning training method: Effects of various treatments for pruning were tested. Three treatments were done. Partial pruning was done by pruning all branches except one branch. Partial pruning was done by pruning all branches except four and six branches. Data collection: The vegetative and flowerier details in were collected after 150 day from planting. Experimental design and data analysis: The experiment consisted of 18 treatments (two Biofertilizers T. harzianum$\mathrm{T} 22 \mathrm{x}$ Three growing medium $\mathrm{x}$ three pruning training method) were arranged in a splitplot design, pruning training method as main -plots and Biofertilizers and growing medium as sub-plots. Each treatment contained 4 plants (pots) and replicated four times. 
Data were statistically analyzed using SAS 9 analytical software, and the mean were compared using Duncan Multiple-range check of in $(\mathrm{P}<5 \%)$.

\section{RESULTS AND DISCUSSION}

Table (1) shows the effect of $T$. harzianum, growing medium and pruning training method and their interaction on the plant height. Significant differences were observed for plant height. The effects of Trichoderma application on plant height were statistically significant $(\mathrm{p}<0.05)$. The highest plant height is $(52.31 \mathrm{~cm})$. Growing medium did not significantly affect plant height. While pruning training method on six branches record the highest amount in plant height $(52.29 \mathrm{~cm})$ as shown in Table (1), the interaction between Trichoderma application and growing

Table (1): Effect of T. harzianum, growing medium and pruning training method and their interaction plant height $(\mathrm{cm})$ of Lantana camara plants.

\begin{tabular}{|c|c|c|c|c|c|c|}
\hline \multirow{2}{*}{$\begin{array}{c}T . h . \\
\text { application }\end{array}$} & \multirow{2}{*}{$\begin{array}{l}\text { Growing medium } \\
\text { (G.M.) }\end{array}$} & \multicolumn{3}{|c|}{$\begin{array}{l}\text { Pruning training method } \\
\text { (T.M) }\end{array}$} & \multirow{2}{*}{$\begin{array}{l}\text { T.h. X } \\
\text { G.M. }\end{array}$} & \multirow{2}{*}{$\begin{array}{c}\text { Average } \\
\text { of } T . h .\end{array}$} \\
\hline & & $\begin{array}{c}1 \\
\text { branch }\end{array}$ & $\begin{array}{c}\text { Four } \\
\text { branches }\end{array}$ & $\begin{array}{l}\text { Six } \\
\text { branches }\end{array}$ & & \\
\hline \multirow{3}{*}{ Application } & Soil & $\begin{array}{c}47.83 \\
\text { b-e }\end{array}$ & $\begin{array}{c}56.83 \\
\mathrm{a} \\
\end{array}$ & $\begin{array}{c}52.66 \\
\text { a-d } \\
\end{array}$ & $\begin{array}{c}52.44 \\
\mathrm{~A} \\
\end{array}$ & \multirow{3}{*}{$\begin{array}{c}52.31 \\
\mathrm{~A}\end{array}$} \\
\hline & 1:3 soil: peat moss & $\begin{array}{c}43.66 \\
\mathrm{e}\end{array}$ & $\begin{array}{c}52.58 \\
\text { a-d }\end{array}$ & $\begin{array}{c}59.07 \\
\mathrm{a}\end{array}$ & $\begin{array}{c}51.77 \\
\mathrm{~A}\end{array}$ & \\
\hline & 1:1 soil: peat moss & $\begin{array}{c}45.41 \\
d-f\end{array}$ & $\begin{array}{c}53.66 \\
a-c \\
\end{array}$ & $\begin{array}{c}59.09 \\
\mathrm{a} \\
\end{array}$ & $\begin{array}{c}52.72 \\
\mathrm{~A}\end{array}$ & \\
\hline \multirow{3}{*}{$\begin{array}{l}\text { Without } \\
\text { application }\end{array}$} & Soil & $\begin{array}{c}44.83 \\
\text { d-e }\end{array}$ & $\begin{array}{c}47.16 \\
b-e\end{array}$ & $\begin{array}{c}48.83 \\
b-e\end{array}$ & $\begin{array}{c}46.75 \\
\text { B }\end{array}$ & \multirow{3}{*}{$\begin{array}{c}48.25 \\
\text { B }\end{array}$} \\
\hline & 1:3 soil: peat moss & $\begin{array}{c}47.58 \\
b-c\end{array}$ & $\begin{array}{l}46.25 \\
\text { c-e }\end{array}$ & $\begin{array}{c}46.41 \\
\mathrm{c}-\mathrm{e}\end{array}$ & $\begin{array}{c}47.75 \\
\text { B }\end{array}$ & \\
\hline & 1:1 soil: peat moss & $\begin{array}{c}55.16 \\
\mathrm{ab} \\
\end{array}$ & $\begin{array}{l}47.33 \\
\mathrm{~b}-\mathrm{e}\end{array}$ & $\begin{array}{c}47.75 \\
b-e\end{array}$ & $\begin{array}{c}50.08 \\
\mathrm{Ab}\end{array}$ & \\
\hline \multirow{2}{*}{$\begin{array}{c}\text { T.h. } \\
\text { X } \\
\text { T.M }\end{array}$} & T.harzianum & $\begin{array}{c}45.63 \\
b\end{array}$ & $\begin{array}{c}54.36 \\
\mathrm{ab}\end{array}$ & $\begin{array}{c}57.33 \\
\mathrm{a} \\
\end{array}$ & \multirow{2}{*}{\multicolumn{2}{|c|}{$\begin{array}{c}\text { Average of } \\
\text { Growing medium }\end{array}$}} \\
\hline & Without T.h. & $\begin{array}{c}49.19 \\
\text { bc }\end{array}$ & $\begin{array}{c}46.91 \\
\mathrm{c} \\
\end{array}$ & $\begin{array}{c}47.66 \\
\mathrm{c}\end{array}$ & & \\
\hline \multirow{3}{*}{$\begin{array}{c}\text { G.M } \\
\text { X } \\
\text { T. M }\end{array}$} & Soil & $\begin{array}{c}46.33 \\
b\end{array}$ & $\begin{array}{c}52.00 \\
\mathrm{a} \\
\end{array}$ & $\begin{array}{c}50.47 \\
\mathrm{ab} \\
\end{array}$ & \multicolumn{2}{|c|}{$\begin{array}{c}50.08 \\
\mathrm{~A}\end{array}$} \\
\hline & 1:3 soil: peat moss & $\begin{array}{c}45.26 \\
b\end{array}$ & $\begin{array}{c}46.41 \\
\mathrm{ab}\end{array}$ & $\begin{array}{c}53.00 \\
\mathrm{a} \\
\end{array}$ & \multicolumn{2}{|c|}{$\begin{array}{c}52.42 \\
\mathrm{~A}\end{array}$} \\
\hline & 1:1 soil: peat moss & $\begin{array}{c}50.29 \\
\mathrm{ab}\end{array}$ & $\begin{array}{c}50.50 \\
\mathrm{ab}\end{array}$ & $\begin{array}{c}53.17 \\
\mathrm{a} \\
\end{array}$ & \multicolumn{2}{|c|}{$\begin{array}{c}51.97 \\
\mathrm{~A}\end{array}$} \\
\hline \multicolumn{2}{|c|}{ Average of T.M. } & $\begin{array}{c}47.14 \\
b\end{array}$ & $\begin{array}{c}50.63 \\
\mathrm{ab}\end{array}$ & $\begin{array}{c}52.29 \\
\mathrm{a} \\
\end{array}$ & & \\
\hline
\end{tabular}

Different letters in the columns indicate significant differences $(\mathrm{p}<0.05)$, according to Duncan's new multiple range test. 
media reveals significant increase in plant height of Lantana plants where (Trichoderma application $X$ 1:1 soil: peat moss) record the highest in plant height $(52.72 \mathrm{~cm})$. Moreover, both of pruning training method on 4 and 6 branches recorded the highest significant increase in plant height $(54.36,57.33 \mathrm{~cm})$ when Trichoderma is applied. Finally, the interaction between Trichoderma application, growing media and pruning training method reveals significant increase in plant height of Lantana plants where (Trichoderma application X 1:1 soil: peat moss X pruning training method on 6 branches) record the highest in plant height $(59.09 \mathrm{~cm})$.

Table (2) shows that Trichoderma application do not significantly affect stem diameter. Growing medium significantly affect stem diameter with an average (8.28 mm) With 1:1 soil peat moos.

Training on four branches record the highest amount in stem length $(8.38 \mathrm{~mm})$ as shown in Table (2), the interaction between Trichoderma application and growing media reveal significant increase in stem diameter of Lantana plants where (Trichoderma application $X$ 1:1 soil+ peat moos) record the highest in stem diameter $(8.32 \mathrm{~mm})$. Moreover, Training on 6 branches recorded the highest significant increase in stem diameter $(8.89 \mathrm{~mm})$ when Trichoderma is applied. Finally, the interaction between Trichoderma application, growing media and Training method reveals significant increase in stem diameter of Lantana plants where (Trichoderma application X 1:1 soil+ peat moos $X$ Training on 6 branches) record the highest in stem diameter $(9.40$ $\mathrm{mm})$.

Table (2): Effect of T. harzianum, growing medium and pruning training method and their interaction in stem diameter $(\mathrm{mm})$ of Lantana camara.

\begin{tabular}{|c|c|c|c|c|c|c|}
\hline \multirow{2}{*}{$\begin{array}{c}T . h, \\
\text { application }\end{array}$} & \multirow{2}{*}{$\begin{array}{l}\text { Growing medium } \\
\text { (G.M.) }\end{array}$} & \multicolumn{3}{|c|}{ Pruning training method (T.M) } & \multirow{2}{*}{$\begin{array}{l}\text { T.h. X } \\
\text { G. M. }\end{array}$} & \multirow{2}{*}{$\begin{array}{c}\text { Average } \\
\text { of } T . h .\end{array}$} \\
\hline & & $\begin{array}{c}1 \\
\text { branch }\end{array}$ & $\begin{array}{c}\text { Four } \\
\text { Branche } \\
\text { s }\end{array}$ & $\begin{array}{l}\text { Six } \\
\text { branches }\end{array}$ & & \\
\hline \multirow[t]{3}{*}{ Application } & Soil & $7.24 \mathrm{c}-\mathrm{e}$ & $7.56 \mathrm{~b}-\mathrm{e}$ & $8.00 \mathrm{a}-\mathrm{d}$ & $7.60 \mathrm{ab}$ & \multirow[t]{3}{*}{$8.06 \mathrm{a}$} \\
\hline & 1:3 soil: peat moss & $7.07 \mathrm{c}-\mathrm{e}$ & $8.79 a-c$ & $9.08 \mathrm{a} \mathrm{b}$ & $8.31 \mathrm{a}$ & \\
\hline & 1:1 soil: peat moss & $7.10 \mathrm{c}-\mathrm{e}$ & $8.32 \mathrm{a}-\mathrm{c}$ & $9.40 \mathrm{a}$ & $8.27 \mathrm{a} \mathrm{b}$ & \\
\hline \multirow{3}{*}{$\begin{array}{l}\text { Without } \\
\text { application }\end{array}$} & Soil & $6.56 \mathrm{~d}-\mathrm{e}$ & $8.48 \mathrm{a}-\mathrm{c}$ & $8.12 \mathrm{a}-\mathrm{d}$ & $7.72 \mathrm{ab}$ & \multirow[t]{3}{*}{$7.82 \mathrm{a}$} \\
\hline & 1:3 soil: peat moss & $6.04 \mathrm{e}-$ & $8.59 a-c$ & $7.67 \mathrm{a}-\mathrm{e}$ & $7.43 \mathrm{~b}$ & \\
\hline & 1:1 soil: peat moss & $8.51 \mathrm{a}-\mathrm{c}$ & $8.57 \mathrm{a}-\mathrm{c}$ & $7.90 \mathrm{a}-\mathrm{d}$ & $8.32 \mathrm{a}$ & \\
\hline \multirow{2}{*}{$\begin{array}{l}\text { T.h, X } \\
\text { T.M. }\end{array}$} & T.harzianum & $7.14 \mathrm{~b}$ & $8.25 \mathrm{a}$ & $8.71 \mathrm{a}$ & \multirow{2}{*}{\multicolumn{2}{|c|}{$\begin{array}{c}\text { Average of } \\
\text { Growing medium }\end{array}$}} \\
\hline & Without T.h. & $7.04 \mathrm{~b}$ & $8.55 b$ & 7.89ab & & \\
\hline \multirow{3}{*}{$\begin{array}{c}\text { G.M. } \\
\text { X } \\
\text { T. M. }\end{array}$} & Soil & $6.90 \mathrm{c}$ & $8.02 \mathrm{a}$ & $8.06 \mathrm{a}$ & \multicolumn{2}{|c|}{$7.64 b$} \\
\hline & 1:3 soil: peat moss & $6.56 \mathrm{c}$ & $8.69 a$ & $8.38 \mathrm{a}$ & \multicolumn{2}{|c|}{$7.89 \mathrm{ab}$} \\
\hline & 1:1 soil: peat moss & $7.81 \mathrm{a} \mathrm{b}$ & $8.45 a$ & $8.47 \mathrm{a}$ & \multicolumn{2}{|c|}{$8.28 \mathrm{a}$} \\
\hline \multicolumn{2}{|c|}{ Average of T. M. } & $7.09 \mathrm{~b}$ & $8.38 \mathrm{a}$ & $8.30 \mathrm{a}$ & & \\
\hline
\end{tabular}

Different letters in the columns indicate significant differences $(\mathrm{p}<0.05)$, according to Duncan's new multiple range test.

Table (3) Leaf number per plant was high in Lantana plants treated with Trichoderma in all Training method compared to untreated plants (Table 3). Peat moos growing medium in 1:1 significantly affect Leif number in all Training method compared to plants growing in soil 1:3 (Table 3). The interaction between Trichoderma 
application and growing media do not reveal significant increase in Leif number of Lantana plants which are trained on one branch. while it is significant in Training on 4 and 6 branches (Table 3).

Table (3): Effect of T. harzianum, growing medium and pruning training method and their interaction in Leif number per plant of Lantana camara.

\begin{tabular}{|c|c|c|c|c|c|c|}
\hline \multirow{2}{*}{$\begin{array}{c}T . h, \\
\text { Applicati } \\
\text { on }\end{array}$} & \multirow{2}{*}{$\begin{array}{l}\text { Growing medium } \\
\text { (G.M.) }\end{array}$} & \multicolumn{3}{|c|}{ Pruning training method (T.M) } & \multirow{2}{*}{$\begin{array}{l}\text { T.h. X } \\
\text { G. M. }\end{array}$} & \multirow{2}{*}{$\begin{array}{l}\text { Average } \\
\text { of T.h. }\end{array}$} \\
\hline & & $\begin{array}{c}1 \\
\text { branch }\end{array}$ & $\begin{array}{c}\text { Four } \\
\text { Branches }\end{array}$ & $\begin{array}{c}\text { Six } \\
\text { branches }\end{array}$ & & \\
\hline \multirow{3}{*}{$\begin{array}{l}\text { Applicati } \\
\text { on }\end{array}$} & Soil & $16.75 a$ & $92.40 \mathrm{~b} \mathrm{c}$ & $122.75 d$ & $77.30 \mathrm{a}$ & \multirow[t]{3}{*}{$101.91 \mathrm{a}$} \\
\hline & 1:3 soil: peat moss & $16.00 \mathrm{a}$ & $120.33 c$ & $180.33 \mathrm{ab}$ & $105.55 \mathrm{a}$ & \\
\hline & 1:1 soil: peat moss & $16.58 \mathrm{a}$ & $160.67 \mathrm{a}$ & $191.44 \mathrm{a}$ & $122.89 \mathrm{a}$ & \\
\hline \multirow{3}{*}{$\begin{array}{c}\text { Without } \\
\text { applicatio } \\
n\end{array}$} & Soil & $17.80 \mathrm{a}$ & $98.09 \mathrm{~d}$ & $171.08 \mathrm{~b}$ & $95.65 \mathrm{~b}$ & \multirow[t]{3}{*}{$95.38 \mathrm{a}$} \\
\hline & 1:3 soil: peat moss & $15.90 \mathrm{a}$ & $134.36 \mathrm{ab}$ & $156.83 c$ & $102.36 \mathrm{a}$ & \\
\hline & 1:1 soil: peat & $15.33 \mathrm{a}$ & $114.62 \mathrm{ab}$ & $134.50 \mathrm{~d}$ & $88.15 \mathrm{~b}$ & \\
\hline \multirow{2}{*}{$\begin{array}{l}\text { T.h, X } \\
\text { T.M. }\end{array}$} & T.harzianum & $16.44 \mathrm{c}$ & $124.47 \mathrm{~b}$ & $164.84 \mathrm{a}$ & \multirow{2}{*}{\multicolumn{2}{|c|}{$\begin{array}{c}\text { Average of } \\
\text { Growing medium }\end{array}$}} \\
\hline & Without T.h. & $24.52 \mathrm{c}$ & $115.69 \mathrm{~b}$ & $154.14 \mathrm{a}$ & & \\
\hline \multirow{3}{*}{$\begin{array}{c}\text { G.M. } \\
\text { X } \\
\text { T. M. }\end{array}$} & Soil & $17.28 \mathrm{~d}$ & $95.25 \mathrm{c}$ & $151.75 b$ & \multicolumn{2}{|c|}{$88.09 \mathrm{~b}$} \\
\hline & 1:3 soil: peat moss & $15.95 \mathrm{~d}$ & $127.35 \mathrm{~b}$ & $164.66 \mathrm{a}$ & \multicolumn{2}{|c|}{$102.65 \mathrm{a}$} \\
\hline & 1:1 soil: peat moss & $15.95 d$ & $137.65 \mathrm{~b}$ & $158.90 \mathrm{ab}$ & \multicolumn{2}{|c|}{$104.17 \mathrm{a}$} \\
\hline \multicolumn{2}{|c|}{ Average of T. M. } & $20.48 \mathrm{c}$ & $120.02 \mathrm{~b}$ & $159.49 \mathrm{a}$ & & \\
\hline
\end{tabular}

Different letters in the columns indicate significant differences $(\mathrm{p}<0.05)$, according to Duncan's new multiple range test.

The chlorophyll relatives of Lantana plants that measured by SPAD meter is high in Lantana plants inoculated with Trichoderma (25.98 SPAD) compared with untreated plant (23.64 SPAD) (Table 4). Growing medium significantly affect chlorophyll amount with an average (26.29 SPAD). With1:1 soilt peat moos. Training on six branches record the highest amount in chlorophyll (38.69 SPAD) as shown in Table (4), the interaction between Trichoderma application and growing media reveal significant increase in chlorophyll amount in Lantana plants where (Trichoderma application X 1:1 soil+peat moos) record the highest chlorophyll amount (27.19 SPAD). Moreover, Training on 6 branches recorded the highest significant increase in chlorophyll amount (42.41 SPAD) when Trichoderma is applied. Finally, the interaction between Trichoderma application, growing media and Training method reveal significant increase in chlorophyll amount Lantana plants where (Trichoderma application X 1:3 soil+peat moos $\mathrm{X}$ Training on 6 branches) record the highest in chlorophyll amount (44.18 SPAD).

Trichoderma application significantly affect flowering period of Lantana plants with an average (85.84 day. Growing medium also significantly affect flowering period with an average (93.33 day) with 1:1 soil+peat moos (Table 5). While Training methods do not significantly affect flowering period. As shown in Table (5), the interaction between Trichoderma application and growing media reveals significant increase in flowering period of Lantana plants where (Trichoderma application X 1:1 
Table (4): Effect of T. harzianum, growing medium and pruning training method and their interaction in chlorophyll relatives SPAD of Lantana camara.

\begin{tabular}{|c|c|c|c|c|c|c|}
\hline \multirow{2}{*}{$\begin{array}{c}\text { T.h. } \\
\text { application }\end{array}$} & \multirow{2}{*}{$\begin{array}{c}\text { Growing medium } \\
\text { (G.M) }\end{array}$} & \multicolumn{3}{|c|}{ Pruning training method (T.M) } & \multirow{2}{*}{$\begin{array}{l}\text { T.h. X } \\
\text { G. M. }\end{array}$} & \multirow{2}{*}{$\begin{array}{c}\text { Average } \\
\text { of } T . h .\end{array}$} \\
\hline & & $\begin{array}{c}1 \\
\text { branch }\end{array}$ & $\begin{array}{c}\text { Four } \\
\text { Branches }\end{array}$ & $\begin{array}{c}\text { Six } \\
\text { branches }\end{array}$ & & \\
\hline \multirow[t]{3}{*}{ Application } & Soil & $13.46 \mathrm{ef}$ & $23.27 \mathrm{c} \mathrm{d}$ & $39.38 \mathrm{ab}$ & $25.37 \mathrm{~b}$ & \multirow[t]{3}{*}{$25.98 \mathrm{a}$} \\
\hline & 1:3 soil: peat moss & $14.56 \mathrm{ef}$ & $17.47 \mathrm{df}$ & $44.18 \mathrm{a}$ & $25.4 \mathrm{~b}$ & \\
\hline & 1:1 soil: peat moss & $24.85 \mathrm{~cd}$ & $13.05 \mathrm{ef}$ & $43.68 \mathrm{a}$ & $27.19 \mathrm{a}$ & \\
\hline \multirow{3}{*}{$\begin{array}{c}\text { Without } \\
\text { application }\end{array}$} & Soil & $12.67 \mathrm{f}$ & $18.25 \mathrm{de}$ & $35.26 \mathrm{~b}$ & $22.06 \mathrm{c}$ & \multirow[t]{3}{*}{$23.64 \mathrm{~b}$} \\
\hline & 1:3 soil: peat moss & $15.97 \mathrm{ef}$ & $18.23 \mathrm{de}$ & $36.24 \mathrm{~b}$ & $23.48 \mathrm{bc}$ & \\
\hline & 1:1 soil: peat moss & $21.51 \mathrm{~cd}$ & $21.24 \mathrm{~cd}$ & $33.45 \mathrm{bc}$ & $25.4 \mathrm{bc}$ & \\
\hline \multirow{2}{*}{$\begin{array}{l}\text { T.h. X } \\
\text { T.M. }\end{array}$} & T.harzianum & $17.62 \mathrm{c}$ & $17.93 \mathrm{c}$ & $42.41 \mathrm{a}$ & \multirow{2}{*}{\multicolumn{2}{|c|}{$\begin{array}{c}\text { Average of } \\
\text { Growing medium }\end{array}$}} \\
\hline & Without T.h. & $17.72 \mathrm{c}$ & $19.24 \mathrm{c}$ & $34.98 \mathrm{~b}$ & & \\
\hline \multirow{3}{*}{$\begin{array}{c}\text { G.M. } \\
\text { X } \\
\text { T. M. }\end{array}$} & Soil & $13.06 \mathrm{~d}$ & $26.76 \mathrm{~b}$ & $37.23 \mathrm{ab}$ & \multicolumn{2}{|c|}{$25.68 \mathrm{a}$} \\
\hline & 1:3 soil: peat moss & $15.26 \mathrm{~cd}$ & $17.85 \mathrm{c}$ & $40.21 \mathrm{a}$ & \multicolumn{2}{|c|}{$18.6 \mathrm{~b}$} \\
\hline & 1:1 soil: peat moss & $23.18 \mathrm{~b}$ & $17.14 \mathrm{e}$ & $38.56 \mathrm{ab}$ & \multicolumn{2}{|c|}{$26.29 \mathrm{a}$} \\
\hline \multicolumn{2}{|c|}{ Average of T. M. } & $17.67 \mathrm{c}$ & $18.58 \mathrm{~b}$ & $38.69 \mathrm{a}$ & & \\
\hline
\end{tabular}

Different letters in the columns indicate significant differences $(\mathrm{p}<0.05)$, according to Duncan's new multiple range test.

soil + peat moos) record the highest in flowering period (100.17 day). Moreover, both of Training on 1 and 6 branches recorded the highest significant increase flowering period (94.33, 90.91 day) when Trichoderma is applied. Finally, the interaction between Trichoderma application, growing media and Training method reveal

Table (5): Effect of T. harzianum, growing medium and pruning training method and their interaction in flowering period (day) of Lantana camara.

\begin{tabular}{|c|c|c|c|c|c|c|}
\hline \multirow{2}{*}{$\begin{array}{c}\text { T.h. } \\
\text { applicati } \\
\text { on }\end{array}$} & \multirow{2}{*}{$\begin{array}{l}\text { Growing medium } \\
\text { (G.M.) }\end{array}$} & \multicolumn{3}{|c|}{ Pruning training method (T.M) } & \multirow{2}{*}{$\begin{array}{l}\text { T.h. X } \\
\text { G. M. }\end{array}$} & \multirow{2}{*}{$\begin{array}{c}\text { Averag } \\
\text { e of } \\
\text { T.h. }\end{array}$} \\
\hline & & $\begin{array}{c}1 \\
\text { branch } \\
\end{array}$ & $\begin{array}{c}\text { Four } \\
\text { Branches }\end{array}$ & $\begin{array}{c}\text { Six } \\
\text { branches }\end{array}$ & & \\
\hline \multirow{3}{*}{$\begin{array}{l}\text { Applicati } \\
\text { on }\end{array}$} & Soil & $88.00 \mathrm{~b}-\mathrm{e}$ & $87.92 b-e$ & $32.25 \mathrm{gh}$ & $69.47 \mathrm{c}$ & \multirow[t]{3}{*}{$85.84 a$} \\
\hline & 1:3 soil: peat moss & 95.0a-d & $57.0 \mathrm{e}-\mathrm{g}$ & $11.67 \mathrm{ab}$ & $87.88 b$ & \\
\hline & 1:1 soil: peat moss & $100.0 \mathrm{a}-\mathrm{c}$ & $74.69 \mathrm{c}-\mathrm{d}$ & $125.83 \mathrm{a}$ & $100.17 \mathrm{a}$ & \\
\hline \multirow{3}{*}{$\begin{array}{l}\text { Without } \\
\text { applicati } \\
\text { on }\end{array}$} & Soil & $44.0 \mathrm{f}-\mathrm{h}$ & $24.0 \mathrm{~h}$ & $96.00 \mathrm{a}-\mathrm{d}$ & $45.66 \mathrm{~d}$ & \multirow[t]{3}{*}{$68.09 b$} \\
\hline & 1:3 soil: peat moss & $43.17 \mathrm{f}-\mathrm{h}$ & $108.0 \mathrm{ab}$ & $61.0 \mathrm{e}-\mathrm{g}$ & $70.72 \mathrm{c}$ & \\
\hline & 1:1 soil: peat moss & $67.0 \mathrm{~d}-\mathrm{f}$ & $117.0 \mathrm{ab}$ & $75.5 \mathrm{c}-\mathrm{d}$ & $86.50 \mathrm{~b}$ & \\
\hline \multirow{2}{*}{$\begin{array}{l}\text { T.h. X } \\
\text { T.M. }\end{array}$} & T.harzianum & $94.17 \mathrm{a}$ & $73.63 b$ & $90.91 \mathrm{ab}$ & \multirow{2}{*}{\multicolumn{2}{|c|}{$\begin{array}{c}\text { Average of } \\
\text { Growing medium }\end{array}$}} \\
\hline & Without T.h. & $51.39 \mathrm{c}$ & $83.00 \mathrm{ab}$ & $77.50 \mathrm{ab}$ & & \\
\hline \multirow{3}{*}{$\begin{array}{l}\text { G.M. X } \\
\text { T.M. }\end{array}$} & Soil & $66.00 \mathrm{f}$ & $55.69 \mathrm{~h}$ & $50.75 \mathrm{~g}$ & \multicolumn{2}{|c|}{$62.91 \mathrm{c}$} \\
\hline & 1:3 soil: peat moss & $69.09 \mathrm{e}$ & $82.50 \mathrm{~d}$ & $86.33 \mathrm{c}$ & \multicolumn{2}{|c|}{$79.30 \mathrm{~b}$} \\
\hline & 1:1 soil: peat moss & $83.50 \mathrm{~d}$ & $95.84 b$ & $100.66 \mathrm{a}$ & \multicolumn{2}{|c|}{$93.33 \mathrm{a}$} \\
\hline \multicolumn{2}{|c|}{ Average of T. M. } & $72.55 \mathrm{a}$ & $78.10 \mathrm{a}$ & $79.25 \mathrm{a}$ & & \\
\hline
\end{tabular}

Different letters in the columns indicate significant differences $(\mathrm{p}<0.05)$, according to Duncan's new multiple range test. 
significant increase in flowering period of Lantana plants where Trichoderma application X 1:1 soil+peat moos X Training on 6 branches) record the highest in flowering period (125.83 day).

\section{DISCUSSION}

Although Trichoderma is known mainly as soil fungi that are widely distributed in the natural environment establish symbioses with several plants (Halifu et al., 2019). The interaction of plant rhizosphere with soil microorganisms plays a major role in soil plant growth, yield, nutrient cycling, and energy conversion, and it is known as a bio control agent, and also functions as plant growth promoting (Rubio et al., 2012; Vinale et al., 2013 and Shoresh et al., 2010). Trichoderma spp. iIs able to increase plant resistance under suboptimal growth conditions, nutrient absorption efficiency, photosynthetic efficiency, root and aboveground plant parts production, increase root hair formation and improve deeper rooting (Lorito et al., 2010 and Shoresh et al., 2010). Plant root exudates facilitate colonization of microorganisms in the rhizosphere, while soil microorganisms enhance plant growth and increase the amount of nutrients in the soil environment by making effective use of plant photosynthesis (Halifu et al., 2019). Trichoderma spp. has beneficial effects can be divided into direct and indirect beneficial effects. The direct beneficial effects include the promotion of plant growth, the promotion and enhancement of plant root growth and structure, the enhancement of seed vigor and development and the decomposition, recycling and use of soil nutrients. (Harman, 2006), showed that the inoculation of Trichoderma spp. In maize, plant production, shifting root structure and growing root activity may be important. Trichoderma may be used as an organic fertilizer as a growth substratum to degrade soil nutrients and enhance plant photosynthesis efficiency, thus improving plant growth. IAA is a plant- hormone and a few microbes synthesized this molecule. IAA plays a key role in the development of root and shoot at plants. Via different transporter system, the hormone travels from one part of the plant to another. Trichoderma treatments improved dramatically relative chlorophyll content of $B$. juncea Plants of as compared to control. (Harman and Shoresh, 2007). Peat moss significantly increased leaves area of petunia. substratum which contains peat moss affects the size of leaves of Antirrhinum majus L. (Mehmood et al., 2013). This, could be because of the high nutrient availability in growing substrates during period the vegetative and reproductive growth periods. results also showed that growing media produced significant effect on number and length of lateral branch of potted petunia plants. Cocopeat increased the number of lateral branches as compared to the other treatments (Olle et al., 2012).

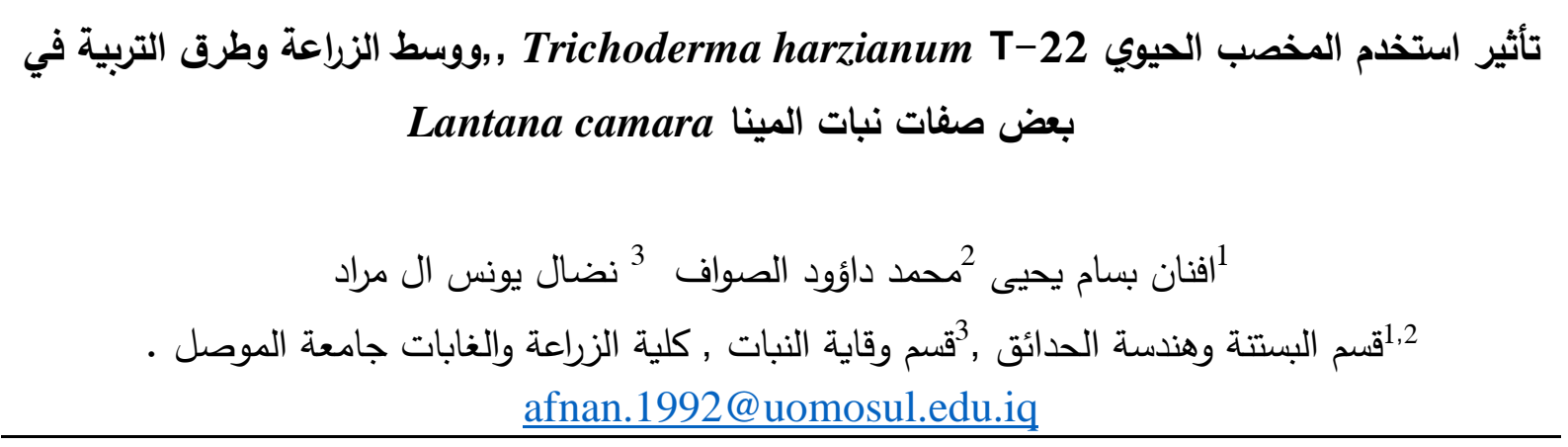




\section{الخلاصة}

اجريت هذه الدراسة في الظلة الثبكية التابعة لقسم البستنة وهندسة الحدائق في كلية الزراعة والغابات جامعة الموصل للمدة من أيار 2019 ولغاية تشرين الأول 2019، بهدف دراسة دور المخصب الحيوي Trichoderma بمسـتويين إضـافة او بدون إضـافة وثلاثة أوسـاط للزراعة وهي زميج نهري، 3:1 زميج نهري+ بتموس، 1:1 زميج نهري + بتموس وثلاثة طرق للتربية بفرع واحد، 4 افرع، 6 افرع وتداخلاتها في في

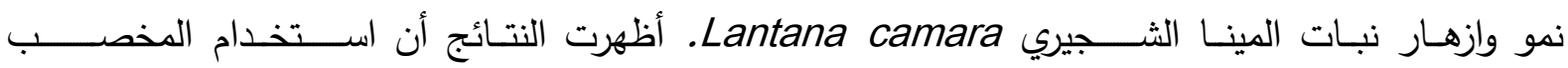
الحيوي T.harzianum مع وســــ النمو وطريقة التربية كان لها تأثيرات كبيرة على النمو الخضــــري والإزهار و سجلت أعلى متوسط لارتفاع النبات (52.31 سم) وقطر السـاق (8.06 ملم) وعدد الأوراق (164.65) وشدة

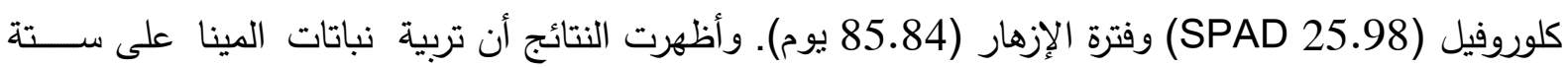
فروع أعطى أعلى متوســــ لارتفاع النبات (52.29 ســـم) وشــــة الكلوروفيل (SPAD 38.69) فترة إزهار

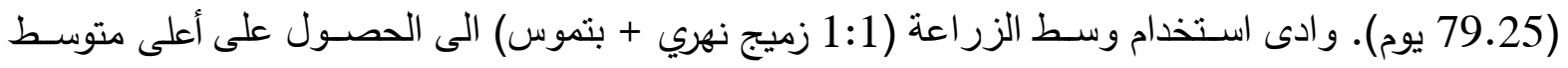

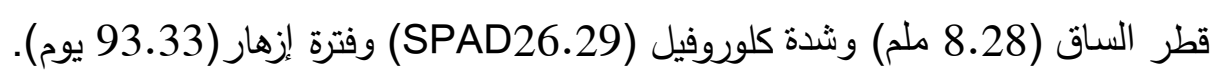

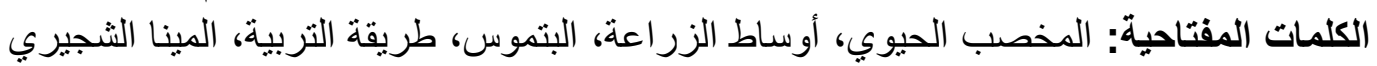
تاريخ تسلم البحث:2020/8/10، وقبوله:2021/3/30

\section{REFERENCES}

Caballero, R., Ordovas,J., Pajuelo,P., Carmona,E., \& Delgado,A. (2007). Iron chlorosis in gerbera as related to properties of various types of compost used as growing media. Communications in Soil Science and Plant Analysis, 38: 2357-2369.

Chong, C. (2005). Experiences with wastes and composts in nursery substrates. Hort Technology, 15:739-747.

Halifu, S., X. Deng, X. Song and R. Song. (2019). Effects of two Trichoderma strains on plant growth, rhizosphere soil nutrients, and fungal community of Pinus sylvestris var. mongolica annual seedlings. Forests, 10(9):1-17. https://doi.org/10.3390/f10090758

Harman, G.E. (2006). Overview of mechanisms and uses of Trichoderma spp. Phytopathology, 96(2):190-194.

Harman, G.E. \& Shoresh,M.(2007). The mechanisms and applications of symbiotic opportunistic plant symbionts. In Novel biotechnologies for biocontrol agent enhancement and management $\mathrm{P}: 131-155$.

Lorito, M., Woo,S.L., Harman,G.E., \& Monte,E. (2010). Translational research on Trichoderma: From 'omics to the field. Annal Review Phytopathology, 48:395-417.

Mazhabi, M., Nemati,S., Rouhani,H., Tehranifar, A., Mahdikhani,M.E., \& Kaveh,H. (2011). How may Trichoderma application affect vegatative and qualitative traits in tulip (Darwin hybride) cultivar. Journal of Biological and Environmental Sciences, 5(15):177-182.

Mehmood, T., Ahmad,W., Ahmad,K.S., Shafi,J., Shehzad,M.A., \& Sarwar,M.A. (2013). Comparative effect of different potting media on vegetative and reproductive growth of floral shower (Antirrhinum majus L.). Universal Journal of Plant Science, 1(3):104-111. https://doi.org/10.13189/ujps. 2013. 010308

Muthahara, E., E. Sitawati and A. Suryanto. (2018). The effect of light intensity and 
paclobutrazol on flowering of Lantana plants (Lantana camara L.). Russian Journal of Agricultural and Socio-Economic Sciences, 80(8):447-451. https://doi.org/10.18551/rjoas.2018-08.60

Olle, M., Ngouajio,M., \& Siomos,A. (2012). Vegetable quality and productivity as influenced by growing medium: A review. Zemdirbyste, 99(4):399-408.

Ostos, J.C., Garrido, R.L., Murillo, J.M., \& Lopez, R.(2008). Substitution of peat for municipal solid-based composts in nursery growing media: Effect on growth and nutrition of the native shrub Pistacia lentiscus. Bioresearch Technology, 99:17931800 .

Rubio, M.B., Domínguez,S., Monte, E., \& Hermosa,R. (2012). Comparative study of Trichoderma gene expression in interactions with tomato plants using highdensity oligonucleotide microarrays. Microbiology, 158(1):119-128. https://doi.org/10.1099/mic.0.052118-0

Saifuddin, M., Hossain, A. B. M. S., Osman, N., Sattar, M. A., Moneruzzaman, K. M., $\&$ Jahirul, M. I. (2010). Pruning impacts on shoot-root-growth, biochemical and physiological changes of'Bougainvillea glabra'. Australian Journal of Crop Science, 4(7), 530-537.

Shoresh, M., Harman,G.E., \& Mastouri,F. (2010). Induced systemic resistance and plant responses to fungal biocontrol agents. Annual review of phytopathology, 48:21-43.

Swain, H., Adak, T., Mukherjee, A. K., Mukherjee, P. K., Bhattacharyya, P., Behera, S., ... \& Jena, M. (2018). Novel Trichoderma strains isolated from tree barks as potential biocontrol agents and biofertilizers for direct seeded rice. Microbiological research, 214, 83-90.

Vinale, F., Nigro, M., Sivasithamparam, K., Flematti, G., Ghisalberti, E. L., Ruocco, M., ... \& Lorito, M. (2013). Harzianic acid: a novel siderophore from Trichoderma harzianum. FEMS microbiology letters, 347(2), 123-129.

Younis, A., Riaz, A., Javaid, F., Ahsan, M., Tariq, U., Aslam, S., \& Majeed, N. (2015). Influence of various growing substrates on growth and flowering of potted miniature rose cultivar “'Baby Boomer'. Curr Sci Perspect, 1, 16-21. 